\title{
Formation of a Direct Kolmogorov-Like Cascade of Second-Sound Waves in He II
}

\author{
G. V. Kolmakov, ${ }^{1,2}$ V. B. Efimov, ${ }^{1,2}$ A. N. Ganshin, ${ }^{1}$ P. V. E. McClintock, ${ }^{1}$ and L. P. Mezhov-Deglin ${ }^{2}$ \\ ${ }^{1}$ Department of Physics, Lancaster University, Lancaster LA1 4YB, United Kingdom \\ ${ }^{2}$ Institute of Solid State Physics RAS, Chernogolovka, Moscow Region 142432, Russia
}

(Received 17 July 2006; published 13 October 2006)

\begin{abstract}
Based on measurements of nonlinear second-sound resonances in a high-quality resonator, we have observed a steady-state wave energy cascade in He II involving a flux of energy through the spectral range towards high frequencies. We show that the energy balance in the wave system is nonlocal in $K$ space and that the frequency scales of energy pumping and dissipation are widely separated. The wave amplitude distribution follows a power law over a wide range of frequencies. Numerical computations yield results in agreement with the experimental observations. We suggest that second-sound cascades of this kind may be useful for model studies of acoustic turbulence.
\end{abstract}

DOI: 10.1103/PhysRevLett.97.155301

Turbulence in the system of waves in a nonlinear nondispersive medium is usually referred to as acoustic turbulence, or Burgers turbulence (BT) in honor of the Burgers equation which provides a useful description of the phenomenon. BT has been at the focus of numerous investigations during the last few decades because of its importance for basic nonlinear physics and in view of numerous applications in engineering and fundamental science [1-3]. Well-known examples of BT include the turbulence of sound waves in oceanic waveguides [4], magnetic turbulence in interstellar gases [5], and shock waves in the solar wind and their coupling with Earth's magnetosphere [6].

In this Letter we propose a novel approach to the experimental study of BT: the use of nonlinear second-sound standing waves in superfluid ${ }^{4} \mathrm{He}$ (He II) within a highquality resonator. Second sound is a slightly dissipative temperature or entropy wave that arises in superfluids and perfect crystals [7]. Second sound in He II is characterized by nonlinear properties that are rather strong compared to those of ordinary sound (pressure or density waves) in conventional solids, liquids, and gases [8-11]. The velocity $u_{2}$ of second sound depends on its amplitude and, to a first approximation, can be written as

$$
u_{2}=u_{20}(1+\alpha \delta T) \text {, }
$$

where $u_{20}$ is the wave velocity at negligibly small amplitude, $\delta T$ is the wave amplitude, and

$$
\alpha=\frac{\partial}{\partial T} \ln \left(u_{20}^{2} \frac{C}{T}\right)
$$

is the nonlinearity coefficient of second sound. $C$ is the heat capacity per unit mass of liquid helium at constant pressure, and $T$ is the temperature.

There are huge advantages in the use of roton secondsound waves in He II for model studies of nonlinear wave interactions. Within the experimentally convenient temperature range $2.17-1.5 \mathrm{~K}$, the nonlinearity coefficient $\alpha$ can be tuned, just by changing the bath temperature: $\alpha \rightarrow$ $-\infty$ near the normal-to-superfluid transition at $T_{\lambda}=$
PACS numbers: 67.40.Pm, 47.27.E-, 47.35.Rs

$2.17 \mathrm{~K} ; \alpha$ passes through zero at $T_{\alpha}=1.88 \mathrm{~K}$; and $\alpha \sim$ $+2 \mathrm{~K}^{-1}$ at $T \sim 1.5 \mathrm{~K}$ [8]. Thus one can study the dynamics of both nearly linear and strongly nonlinear waves with positive (like conventional sound) or negative nonlinearity while using exactly the same experimental techniques. Such possibilities are unavailable in conventional experiments. The fact that the velocity of second sound $u_{20} \leq$ $20 \mathrm{~m} / \mathrm{s}$ is more than an order of magnitude less than the velocity of conventional sound in gases and in condensed media allows us to increase the time resolution of the measurements.

We use a thin-film heater to generate the second sound, and a thin-film thermometer (a low-thermal-inertial superconducting bolometer) as a detector. Use of a high- $Q$ resonator enables us to create nonlinear standing secondsound waves of high amplitude with only small heat production at the source. The wave amplitude $\delta T$ can be changed from $0.05 \mathrm{mK}$ up to a few $\mathrm{mK}$, so that the Mach number can reach $\mathrm{M}=\alpha \delta T \sim 10^{-4}-10^{-2}$, and the proper Reynolds number, defined [12] as $\operatorname{Re}=\alpha u_{20}(\partial \delta T / \partial x) /$ $\gamma_{\omega} \sim \alpha Q \delta T$, can be changed from 1 to 90 (here $\gamma_{\omega}$ is the damping coefficient of a second-sound wave of frequency $\omega$, evaluated from the $Q$ factor of the resonator). It allows us to study, for the first time, the transition from the linear regime to turbulence in the system of second-sound waves as the driving amplitude is increased. The experimental arrangements were similar to those used earlier [10]. The resonator was formed by a cylindrical quartz tube of length $L=7 \mathrm{~cm}$ and inner diameter $D=1.5 \mathrm{~cm}$. The film heater and bolometer were deposited on the surfaces of flat glass plates capping the ends of the tube. The heater was driven by an external sinusoidal voltage generator in the frequency range between 0.1 and $100 \mathrm{kHz}$. The frequency of the second sound (at twice the frequency of the voltage generator) was set equal to one of the longitudinal resonant frequencies. The measurements reported below were conducted at $T=2.079 \mathrm{~K}$ where $\alpha \approx-7.6 \mathrm{~K}^{-1}$, and at $T=$ $1.775 \mathrm{~K}$ where $\alpha \approx 1.8 \mathrm{~K}^{-1}$. The $Q$ factor of the resonator determined from the widths of longitudinal resonances at small heat flux densities was $Q \sim 1000$ for resonant 
numbers $p \leq 10$, and $Q \sim 3000$ for $30<p<100$. The steady-state second-sound distribution in the resonator was Fourier analyzed and its power spectrum was computed.

Figure 1 shows some typical experimental results at $2.079 \mathrm{~K}$. For a small ac heat flux density $W<$ $1 \mathrm{~mW} / \mathrm{cm}^{2}$, corresponding to a standing wave amplitude less than $0.5 \mathrm{mK}$ and to Reynolds numbers $\mathrm{Re} \sim 1$, we observed a nearly linear regime of wave generation: the amplitude $A$ of the standing wave was proportional to the heat flux density $W$ [see Fig. 1(a), inset]. An increase of the excitation above a few $\mathrm{mW} / \mathrm{cm}^{2}(\operatorname{Re}>10)$ led to large deviations from $A \propto W$ and to visible deformation of the initially sinusoidal standing wave, accounted for by the formation of multiple harmonics in its spectrum, as shown in Fig. 1 and the inset. At $W \sim 80 \mathrm{~mW} / \mathrm{cm}^{2}$ we observed a marked reduction of the $Q$ factor to $\sim 500$ for longitudinal
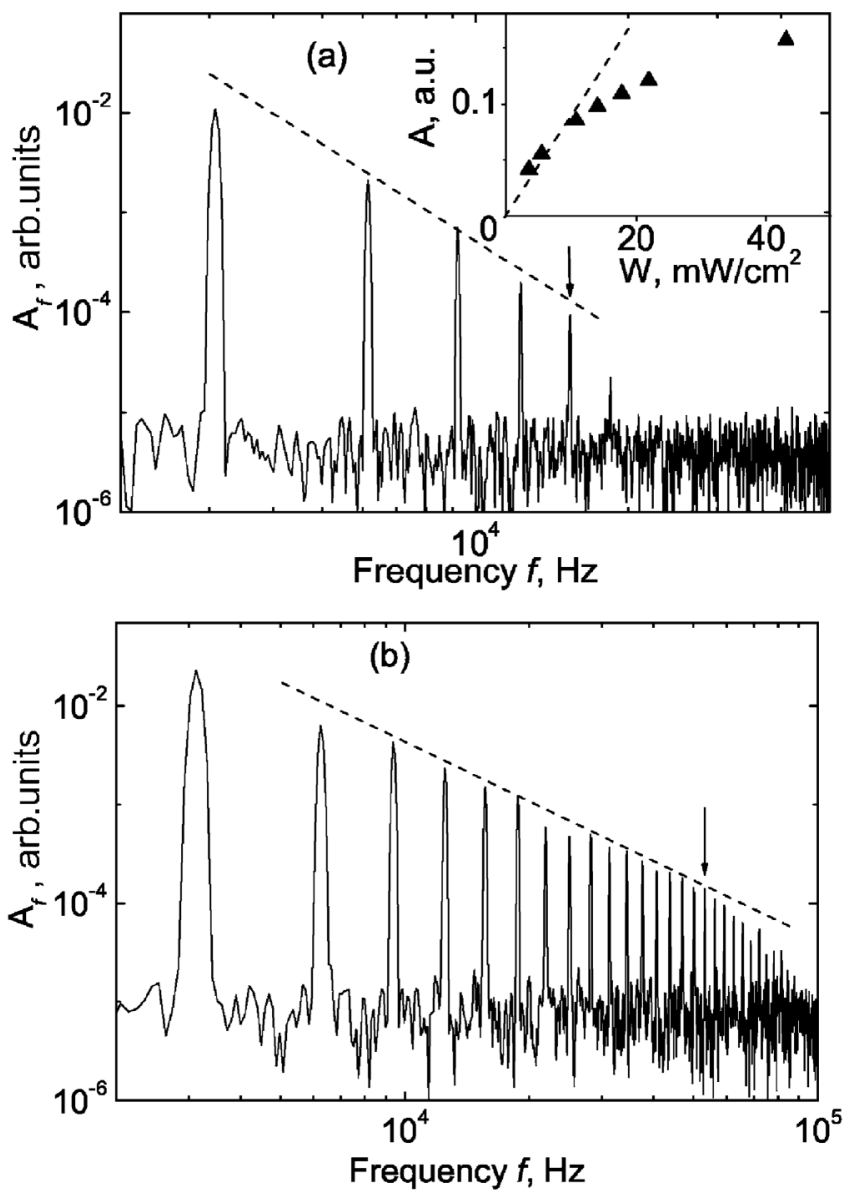

FIG. 1. Power spectral amplitudes $A_{f}$ of standing waves recorded at $T=2.079 \mathrm{~K}$ when driving at the 31 st resonant frequency, $f_{d}=3130 \mathrm{~Hz}$. The ac heat flux density from the heater was (a) $W=5.5$ and (b) $22 \mathrm{~mW} / \mathrm{cm}^{2}$. The dashed line in (a) is a guide to the eye, while the dashed line in (b) corresponds to $A_{f} \propto$ $f^{-1.5}$. The arrows indicate positions of the viscous cutoff frequency $f_{b}$ in each case. In the inset, triangles show the observed dependence of the wave amplitude $A$ on ac heat flux density $W$, measured for 31st resonance, and the dashed line corresponds to the linear dependence $A \propto W$ valid at small $W$. resonances with $p \sim 30$. This may be associated with, e.g., broadening of the resonance curve due to vortex creation in the bulk He II. In what follows, we discuss only the measurements made at $W<80 \mathrm{~mW} / \mathrm{cm}^{2}$.

It is clearly evident from Fig. 1 that the main spectral peak lies at the driving frequency $f_{d}$, and that highfrequency peaks appear at its harmonics, $f_{n}=f_{d} \times n$ with $n=2,3, \ldots$. It can be seen that a cascade of waves is formed over the frequency range up to $15 \mathrm{kHz}$ in Fig. 1(a), and up to $80 \mathrm{kHz}$ in Fig. 1(b), i.e., up to frequencies 5 and 25 times higher than the driving frequency. As shown in Fig. 1(b), the dependence of peak height on frequency may be described by a powerlike law $A_{f}=$ const $\times f_{n}^{-s}$ at frequencies lower than some cutoff frequency $f_{b}$. For sufficiently high heat flux densities $W>$ $10 \mathrm{~mW} / \mathrm{cm}^{2}$, the scaling index tends to $s \approx 1.5 \pm 0.3$.

In order to understand how the wave cascade is formed, we have undertaken a numerical study of the dynamics of nonlinear second-sound waves within the high- $Q$ resonator. Full details will be given elsewhere, but in essence it involves direct integration of the two-fluid thermohydrodynamical equations [7], expanded up to quadratic terms in the wave amplitude. We use a representation of the secondsound waves in terms of Hamiltonian variables [13,14], permitting us to improve the precision of the calculations. The numerical technique is similar to that used in earlier studies [2] of developed two-dimensional acoustic turbulence. In the present model, however, wave damping was taken into account at all frequencies, a feature that is of key importance [15] for a correct description of the formation of a cascade of nonlinear sound waves with increasing driving force amplitude. The numerical results obtained are suitable for direct comparison with our experimental observations shown in Fig. 1.

The equations of motion governing nonlocal energy balance in the system read

$$
\begin{aligned}
i \frac{\partial b_{n}}{\partial t}= & \sum_{n_{1}, n_{2}} V_{n, n_{1}, n_{2}}\left(b_{n_{1}} b_{n_{2}} \delta_{n-n_{1}-n_{2}}+2 b_{n_{1}} b_{n_{2}}^{*} \delta_{n_{1}-n_{2}-n}\right) \\
& -i \gamma_{n} b_{n}+F_{d}
\end{aligned}
$$

where $b_{n}(t)=(1 / 2)\left(B_{n}^{-1} S_{n}+i B_{n} \beta_{n}\right) \quad$ is the timedependent canonical amplitude of second sound at the $n$th resonant frequency $f_{n} ; S_{n}$ and $\beta_{n}$ are the space Fourier components $\sim \cos \left(2 \pi f_{n} x / u_{20} L\right)$ of the entropy and of the potential of the normal-superfluid relative velocity; $B_{n}=\left(f_{n} C / T\right)^{1 / 2} ; V_{n, n_{1}, n_{2}} \propto \alpha\left(n n_{1} n_{2}\right)^{1 / 2}$ describes the three-wave interaction; $\gamma_{n}=\nu n^{2}$ models the viscous damping of second sound; $F_{d} \propto W$ is the amplitude of the force driving the $n$th resonant mode. We neglect the coupling between temperature and pressure oscillations in view of the smallness of the thermal expansion of He II in the relevant temperature range. The wave spectrum was calculated as $A_{f} \propto B_{n}\left(b_{n}+b_{n}^{*}\right)$. We used a periodic driving force of frequency equal to a resonant frequency of the 
resonator corresponding to the conditions of the measurements, taken as $f_{d}=3000$ in dimensionless units. The effective viscosity coefficient $\nu$ was fitted to the measured value of the quality factor $Q \sim 3 \times 10^{3}$ of our resonator, to facilitate comparison of the model results with those from the experiments.

Figure 2 shows the evolution of the steady-state spectrum with increasing driving force amplitude $F_{d}$, calculated for $\alpha<0\left(T>T_{\alpha}\right)$. Points on the plot correspond to the amplitudes of the peaks in the spectrum. It is seen that, at small driving amplitude $F_{d} \sim 0.01$ (triangles), viscous damping prevails at all frequencies and a turbulent cascade is not formed: the amplitude of the second harmonic is an order less than the amplitude of the main harmonic. In this regime the wave shape is close to linear. At intermediate driving amplitude $F_{d} \sim 0.05$ (diamonds), nonlinearity starts to play a role at frequencies of the order of driving frequency, and a few harmonics are generated [cf. Fig. 1(a)]. At high driving amplitudes $F_{d} \geq 0.1$ (circles and squares), a well-developed cascade of second-sound waves is formed up to frequencies 30 times higher than the driving frequency; i.e., the behavior predicted by the computations is very similar to that observed in our experiments [Fig. 1(b)].

Formation of the spectra observed in the experiment and numerics is evidently attributable to the cascade transfer of wave energy through the frequency scales due to nonlinearity, thus establishing an energy flux in $K$ space directed from the driving frequency towards the high-frequency do-

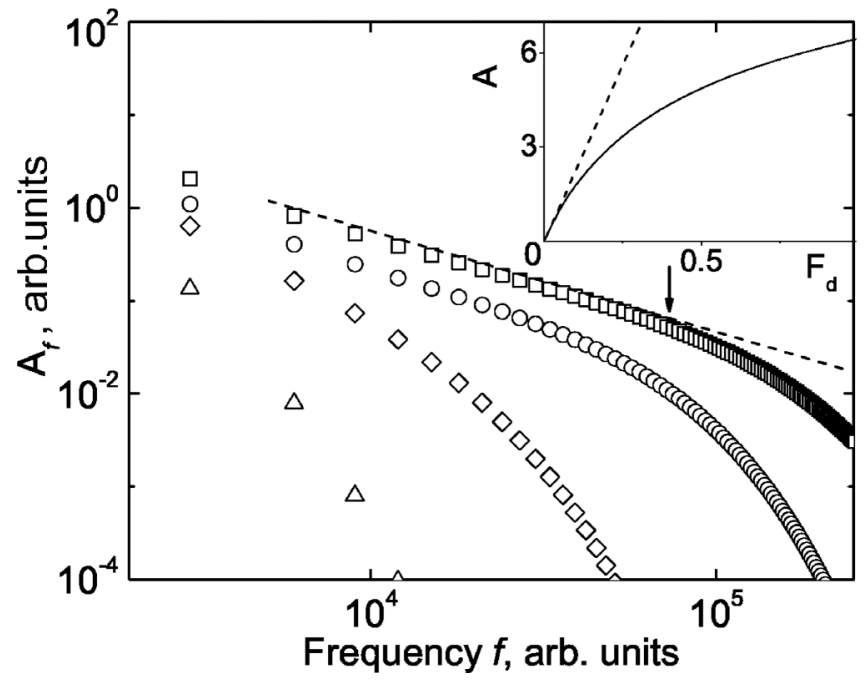

FIG. 2. Second-sound power spectral amplitudes $A_{f}$ calculated numerically from Eq. (2) for four different driving force amplitudes: $F_{d}=0.01$ (triangles), 0.05 (diamonds), 0.1 (circles), and 0.3 (squares). The dashed line corresponds to $A_{f} \propto f^{-1}$ as predicted by the theory $[2,18]$. The arrow marks the boundary frequency $f_{b}$ of the inertial range for $F_{d}=0.3$. The inset shows the calculated dependence on $F_{d}$ of the standing wave amplitude $A$ : solid line, nonlinear waves, $\alpha<0\left(T>T_{\alpha}\right)$; dashed line, linear waves, $\alpha=0\left(T=T_{\alpha}\right)$. main. Following the basic ideas formulated in Refs. [1,1618], such a highly excited state of a system with numerous degrees of freedom is referred to as turbulent. So we may infer that at relatively high driving amplitudes we observe acoustic turbulence formed in the system of second-sound waves within the inertial (nondissipative) range of frequencies. Formation of the observed direct cascade is qualitatively similar to creation of the Kolmogorov distribution of fluid velocities over frequencies in the bulk of classical incompressible fluids [7] or in a system of surface waves $[15,19]$. But we found both in experiment and numerics that the amplitudes of high-frequency harmonics rise gradually with the increase of the driving amplitude.

In some range of frequencies the calculated spectrum obeys a power law with $s \approx 1$ (squares in Fig. 2) that is in qualitative agreement with the theory [2,18]. The difference in slope of the calculated spectrum from the value $s=$ $1.5 \pm 0.3$ observed in the experiment may be attributable to our resonator having an insufficiently high-quality factor, as well as to the influence of other mechanisms of wave energy relaxation not taken into account in our computations as also revealed, e.g., by the appearance of subharmonics when driving at even resonant numbers (see below). The precise identification of the scaling index characterizing a turbulent distribution is obviously important for connecting BT theory to real physical experiments. To clarify the origin of the difference between observations and computations, we plan to make further measurements and to undertake a systematic study in the vicinity of $T_{\lambda}$, where the nonlinearity of second sound rises much faster than its damping [11], and the proper Mach and Reynolds numbers should therefore be substantially increased.

From a comparison of the measurements and computations [Figs. 1(a) and 2, insets] we can conclude also that the deviations from the linear dependence $A \propto W$ observed at moderate driving amplitudes is caused by nonlinear outflow of the energy to higher frequency scales, eventually reaching the dissipative frequency range, rather than by the scattering of second sound from quantized vortices in He II, as considered earlier [11,20].

The effect of the high-frequency cutoff of the wave spectrum manifests itself as an abrupt decrease in the amplitudes of the harmonics at $W<10 \mathrm{~mW} / \mathrm{cm}^{2}$ [cf. Figs. 1(a) and 2 (triangles)], and as a change of slope on double-log scales, at higher $W$ [cf. Figs. 1(b) and 2 (circles and squares)], at some frequency $f=f_{b}$. At $f \sim f_{b}$ the nonlinear mechanism for nearly nondissipative transfer of the wave energy changes to viscous damping of the waves (cf. observations of the high-frequency edge of the inertial range of frequencies of capillary turbulence on the surface of liquid hydrogen [15]). It causes a faster reduction of sound amplitude at frequencies $f>f_{b}$, as observed.

The dependence of the boundary frequency $f_{b}$ on the standing wave amplitude $A$ is shown in Fig. 3. It is clearly evident that the inertial range is extended towards higher frequencies when the driving amplitude is increased. At 


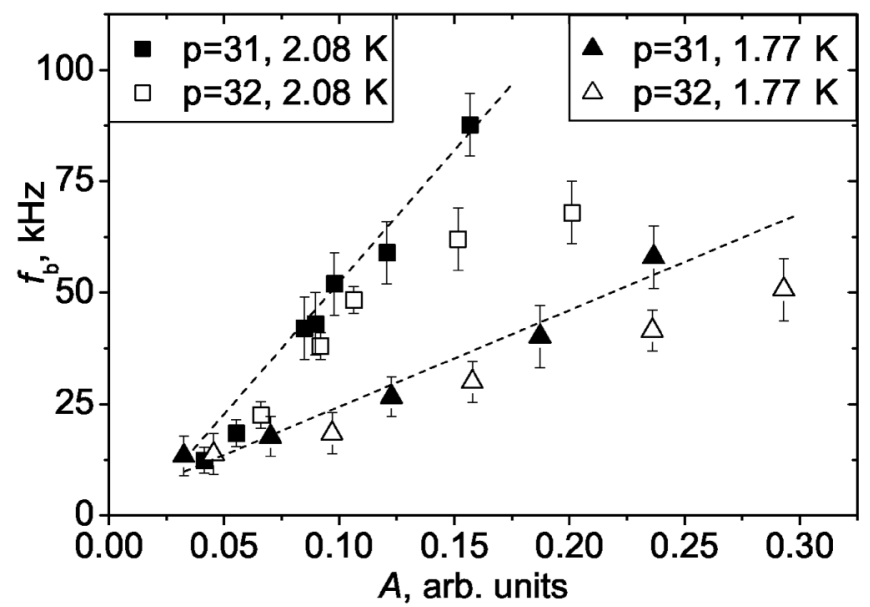

FIG. 3. Dependence of the viscous cutoff frequency $f_{b}$ on the amplitude $A$ of the standing wave for different temperatures, for resonance numbers $p=31$ and 32. Dashed lines, the results of our computations based on the theory [18]; data points, experimental measurements.

sufficiently large amplitudes $A$, the boundary frequency $f_{b}=\operatorname{const}\left(T, f_{d}\right) \times A$, which agrees with our calculations based on [18]. Such a dependence was observed when driving at resonant frequencies with odd resonant numbers $p$ (the solid symbols in Fig. 3).

When driving at even $p$ (open symbols in Fig. 3), however, the boundary frequency is noticeably lower than that measured for the nearest odd resonant number, for $W>$ $10 \mathrm{~mW} / \mathrm{cm}^{2}$. This reduction may be connected with a change in the mechanism of energy relaxation in the wave system caused by the generation of subharmonics with frequencies lower than $f_{d}$. We recently detected the appearance of subharmonics at high $W$ when driving at even $p$, a phenomenon that promises to be of huge interest for nonlinear sound wave dynamics [12,21]. We plan to study it in detail in the near future.

In conclusion, we have demonstrated that the system of roton second-sound waves in a high- $Q$ resonator filled with He II can be used as an effective tool for the detailed modeling and investigation of acoustic turbulence. We observed for the first time a smooth crossover in the system of second-sound waves from a nearly linear regime at low driving amplitudes (at $\mathrm{Re} \sim 1$ ) to a nonlinear regime at moderate driving amplitudes, and, further, to developed turbulence at high driving amplitudes (a Kolmogorovlike cascade [16] at $\operatorname{Re} \sim 10^{2}$ ), rather than a sharp transition to turbulence at Reynolds numbers higher than some critical value as occurs in incompressible fluids [7]. In the high-frequency domain a cutoff of the cascade is observed, caused by a change in the mechanism of energy transfer, from nonlinear wave transformation to viscous damping. Numerical calculations are in qualitative agreement with the results of our experiments.

We are grateful to A. A. Levchenko, V. V. Lebedev, and E. A. Kuznetsov for valuable discussions. The investigations were supported by the Russian Foundation for Basic Research, Projects No. 05-02-17849 and No. 06-02-17253, by the Presidium of the Russian Academy of Sciences under programs "Quantum Macrophysics" and "Mathematical Methods in Nonlinear Dynamics," and by the Engineering and Physical Sciences Research Council (U.K.).

[1] V. E. Zakharov, G. Falkovich, and V. S. L'vov, Kolmogorov Spectra of Turbulence I (Springer, Berlin, 1992).

[2] G. Falkovich and M. Meyer, Phys. Rev. E 54, 4431 (1996).

[3] G. E. Falkovich and A. B. Shafarenko, Sov. Phys. JETP 67, 1393 (1988).

[4] S. N. Gurbatov, V. V. Kurin, L. M. Kustov, and N. V. Pronchatov-Rubtsov, Acoustical Physics 51, 152 (2005).

[5] G. S. Bisnovatyi-Kogan and S. A. Silich, Rev. Mod. Phys. 67, 661 (1995).

[6] M. Ryutova and T. Tarbell, Phys. Rev. Lett. 90, 191101 (2003).

[7] L. D. Landau and E. M. Lifshitz, Course of Theoretical Physics, Fluid Mechanics Vol. 6 (Pergamon, New York, 1987).

[8] A. J. Dessler and W. H. Fairbank, Phys. Rev. 104, 6 (1956).

[9] K. N. Zinov'eva, Sov. Phys. JETP 4, 36 (1957).

[10] I. Yu. Borisenko, V. B. Efimov, and L. P. Mezhov-Deglin, Sov. J. Low Temp. Phys. 14, 619 (1988).

[11] L. S. Goldner, N. Mulders, and G. Ahlers, J. Low Temp. Phys. 93, 131 (1993).

[12] H. Davydowitz, Yu. L'vov, and V. Steinberg, Physica (Amsterdam) 84D, 635 (1995).

[13] V.L. Pokrovskii and I. M. Khalatnikov, Sov. Phys. JETP 44, 1036 (1976).

[14] M. Yu. Brazhnikov et al., Low Temp. Phys. 30, 441 (2004).

[15] G. V. Kolmakov, A. A. Levchenko, M. Yu. Brazhnikov, L.P. Mezhov-Deglin, A. N. Silchenko, and P. V.E. McClintock, Phys. Rev. Lett. 93, 074501 (2004).

[16] A. N. Kolmogorov, Dokl. Akad. Nauk SSSR 30, 299 (1941).

[17] V.E. Zakharov and R.Z. Sagdeev, Dokl. Akad. Nauk SSSR 192, 297 (1970).

[18] E. A. Kuznetsov, JETP Lett. 80, 83 (2004).

[19] A. I. Dyachenko, A. O. Korotkevich, and V.E. Zakharov, Phys. Rev. Lett. 92, 134501 (2004).

[20] L.P. Kondaurova, S. K. Nemirovskii, and M. V. Nedoboiko, J. Low Temp. Phys. 119, 329 (2000).

[21] W. Lauterborn and E. Cramer, Phys. Rev. Lett. 47, 1445 (1981). 